\title{
Autonomous Control of Logistic Processes: A Retrospective
}

\author{
Susanne Schukraft $(\mathbb{D}$, Michael Teucke $\mathbb{( 1 )}$, Michael Freitag (1), \\ and Bernd Scholz-Reiter
}

\begin{abstract}
Manufacturing and logistic service companies are increasingly confronted with high dynamics and complexity. Due to its particular suitability for short-term and situation-dependent decision-making, autonomous control can improve planning and control of production and related transportation processes. This chapter gives an overview of the research that the BIBA-Bremer Institut für Produktion und Logistik $\mathrm{GmbH}$ has performed over the past years in the field of autonomously controlled production and transportation networks. The chapter focuses on the modeling approaches and the autonomous control methods that have been developed. These methods have been evaluated using both theoretical and realworld scenarios. The results show the applicability and suitability of autonomous control in complex and dynamic production and transportation environments. In addition, influences on the methods' performance and the integration of autonomous control into conventional planning and control systems are discussed. Finally, the chapter looks at the significance of autonomous control in the context of Industry 4.0 and shows the relations between both concepts.
\end{abstract}

\footnotetext{
S. Schukraft $(\bowtie) \cdot$ M. Teucke

BIBA - Bremer Institut für Produktion und Logistik $\mathrm{GmbH}$, University of Bremen, Bremen, Germany

e-mail: skf@biba.uni-bremen.de; tck@biba.uni-bremen.de

M. Freitag

BIBA - Bremer Institut für Produktion und Logistik GmbH, University of Bremen, Bremen, Germany

Faculty of Production Engineering, University of Bremen, Bremen, Germany

e-mail: fre@biba.uni-bremen.de

B. Scholz-Reiter

Faculty of Production Engineering, University of Bremen, Bremen, Germany

e-mail: bsr@biba.uni-bremen.de
} 


\section{Introduction}

Over the last decades, manufacturing companies have been confronted with increasing dynamics and complexity (Alkan et al. 2018; Duffie et al. 2017). Among others, companies were challenged with volatile market conditions, decreasing product life cycles, and an increasing product variety (Nyhuis and Wiendahl 2009). The main objective of manufacturing companies is to meet customer requirements regarding product quality and agreed delivery dates (Hon 2005). Therefore, an essential issue for production planning and control is the ability to react flexibly to changing conditions and requirements, e.g., changes in customer demands regarding the product, its volume, or its delivery date. Furthermore, manufacturing companies depend on reliable transportation processes. Thus, to fulfill customer demands, the need for a high degree of flexibility also applies to the related transportation processes for supplying raw materials and delivering finished products to the customer.

Up to now, production planning and control is mainly based on centralized and hierarchical planning approaches. Generally, these approaches calculate production schedules in advance, assuming static production environments. Strategies to cope with changing conditions include the partial or complete rescheduling of the production schedule in the case of occurring demand changes or the provision of robust schedules in advance. However, whereas recurring rescheduling activities may lead to a high planning nervousness, working with robust schedules may be inefficient when the flexibility provided is not utilized (Ouelhadj and Petrovic 2009).

An opposite approach to overcome the limitations of these conventional planning and control approaches is the development of autonomously controlled production systems. Generally, autonomous control is based on decentralized decision-making based on the current system state (Freitag et al. 2004). Thus, autonomous control methods are enabled to flexibly react to changing conditions and achieve a high level of target compliance even in highly complex and dynamic environments.

The BIBA-Bremer Institut für Produktion und Logistik GmbH at the University of Bremen as a member of the Bremen Research Cluster for Dynamics in Logistics (University of Bremen 2021) - has a long history of research in the field of autonomous control of production and logistics, including both manufacturing systems and transportation networks. This paper gives an overview of this research, some of which has also been carried out in collaboration with other research and industrial partners. The presented work mainly bases on the results of the Collaborative Research Center 637 “Autonomous Cooperating Logistic Processes: A paradigm Shift and its Limitations" (CRC 637). The CRC 637 has been carried out at the University of Bremen from 2004 to 2012 with several research departments at the University of Bremen and also the Jacobs University of Bremen. In addition, the research was continued in several follow-up projects that are also considered in the given overview.

This chapter is structured as follows. In Sect. 2, autonomous control and its main characteristics, as well as basic assumptions regarding the performance of 
autonomous control in complex environments, are introduced. In addition, the section discusses the relations between autonomous control and the Industry 4.0 paradigm. An overview of the reference scenarios and modeling approaches for the development of autonomous control methods is given in Sect. 3. Section 4 introduces autonomous control methods developed for either production or transportation scheduling. Section 5 presents the results of various simulation studies mainly based on theoretical reference scenarios. The transfer of autonomous control into real-world applications is described in Sect. 6. A conclusion is given in Sect. 7.

\section{The Concept of Autonomous Control}

Autonomous control is characterized by processes of decentralized decision-making within a heterarchical organization structure. Thus, the decision-making authority is delegated to individual system elements allowed and enabled to make and execute decisions independently on their own (Freitag et al. 2004). In doing so, the logistic objects require the ability to interact with each other to gather relevant decisionmaking information. The characteristics of heterarchy suggest a high degree of independence between individual system elements and the central coordination unit. Furthermore, autonomously controlled systems are characterized by nondeterminism, which means that the system behavior cannot be predicted over a longer period of time, even if all system states are known (Windt and Hülsmann 2007).

The autonomy of logistic objects necessary for decentralized decision-making is realized through information and communication technologies (ICT). The automatic identification of logistic objects requires technologies like barcodes or radiofrequency identification (RFID). Terrestrial technologies can be used for the automatic localization of logistic objects, either satellite-based global navigation satellite systems (GNSS) or long-range, short-range, and indoor terrestrial technologies. Sensor units allow condition monitoring of the logistic objects and their environment. The information processing can be supported by multi-agent systems (MAS) or machine learning algorithms. Technologies like past and recent mobile phone standards or Long Range Wide Area Networks (LoRa) can be used for the information exchange between logistic objects (Böse and Windt 2007b). Altogether, the significant developments in ICT over the last decades that culminate in the introduction of Industry 4.0 can serve as an enabler for implementing autonomously controlled production and logistics systems in practice.

ICT such as RFID or Wi-Fi networks were emerging when the research of the BIBA on developing autonomous control started in 2004 but still not uniformly introduced into the economy. However, it was anticipated that these technologies would gradually become more widely available and used in industry. From 2011 onwards, the concept of Industry 4.0 (Kagermann et al. 2013) emerged and soon established itself internationally as a collective term for the ongoing digitalization and smart automation in industrial production and the integration of the underlying 
technologies. From this moment on, some of the technological developments that were integral to key concepts of autonomous control were subsumed under the generic term Industry 4.0.

Considering the principles of both concepts, it can be stated that autonomous control and Industry 4.0 are closely related to each other. The ability of an autonomous object to process information and render decisions on its own necessitates intelligence embedded into this object in the form of data processor units and sensors that provide data on the object's state and its environment. Also, the interaction between several such autonomous objects requires their ability to exchange data, which presumes them being linked to each other via communication networks. These concepts are closely mirrored by two key principles integral to Industry 4.0, namely decentralized decisions and interconnectivity (Hermann et al. 2016). Decentralized decisions in Industry 4.0 are based on the ability of the socalled cyber-physical systems (CPS). CPS are characterized by the interconnection of information technology and software components with mechanical and electronic parts that communicate via a data infrastructure formed by wired or wireless communication networks (Broy 2010). One example mentioned for decentralized decision-making in Industry 4.0 was products routing themselves through a production system (Kagermann et al. 2013), which was intensively studied in the context of autonomous control (see Sect. 4.2.1). Interconnectivity denotes, in the context of Industry 4.0, the ability of machines, devices, sensors, and people to connect and communicate with each other via the Internet of Things (IoT). The concept of IoT describes the technologies for a global ICT infrastructure that enables the connection of physical and virtual objects and the globally unique identification and addressing of these objects, allowing them to interact and collaborate (Bouhai and Grayson 2017; Chaouchi 2013). In sum, it can be stated that Industry 4.0 provides the technological basis and leads to the widespread use of technical systems that enable autonomously controlled logistics. Autonomous control provides methods and algorithms that can lead to an increase in efficiency in production and logistics, particularly in complex and dynamic environments.

Autonomous control methods take the current system state into account for decision-making. Consequently, these methods are considered especially promising if changing conditions require a flexible reaction of planning and control, e.g., caused by workstation breakdowns or short-term rush orders. However, conventional planning methods are able to create optimal production schedules in advance (i.e., before production orders are released to the shop floor). These methods are often computationally intensive but can outperform autonomous control in static production environments. Furthermore, entirely autonomously controlled production systems also have the risk of a chaotic and unpredictable system's behavior (Windt and Hülsmann 2007). Consequently, potential users of autonomous control will require assistance to assess in which cases and to which extent the application of autonomous control is promising and in which cases conventional planning methods still outperform autonomous control. To address these questions, the following hypotheses were formulated regarding the performance of autonomous 
control. These hypotheses will be revisited below when discussing the methods' performance and comparing them to conventional planning methods.

1. Autonomous control methods are able to cope efficiently with complex and dynamically changing production environments (Freitag et al. 2004).

2. In static production environments, conventional planning and control methods will reach an equal or even better performance than autonomous control. Contrarily, autonomous control will outperform conventional planning and control in environments of high complexity (Philipp et al. 2006).

3. Besides hypothesis (2), the performance of autonomous control methods depends on the present degree of autonomy and the complexity of the applied scenario (Böse and Windt 2007b; Windt et al. 2008). A more detailed description of the assumed interdependencies is given by Philipp et al. (2006).

4. A possibility to combine the advantages of conventional planning and autonomous control - the high planning accuracy of conventional planning in static and the high efficiency of autonomous control in dynamic environmentsis the combined application of both approaches (Schukraft et al. 2016a).

\section{Reference Scenarios for Autonomous Logistic Processes}

This section introduces reference scenarios for both production and transportation scheduling. These theoretical scenarios provide a standardized environment that allows evaluating and comparing the different autonomous control methods' performance. In addition, the section describes the simulation and evaluation of autonomous logistic processes.

\subsection{Description of the Reference Scenarios}

The reference scenarios have been defined in a way that they can easily be adjusted to analyze the influence of various impact factors (e.g., different system sizes or variation of processing times) on the autonomous control methods' performance. Besides, the modeling approaches for the implementation of the autonomous control methods are introduced. Also, this section describes approaches for the measurement of the autonomous control methods' performance.

\subsubsection{Shop-Floor Scenario}

The "NxM shop-floor scenario" is based on a flexible flow shop system. It consists of $\mathrm{M}$ stages, each with $\mathrm{N}$ parallel workstations and an input buffer in front of each workstation. Production orders have to be processed on each stage on one of the 

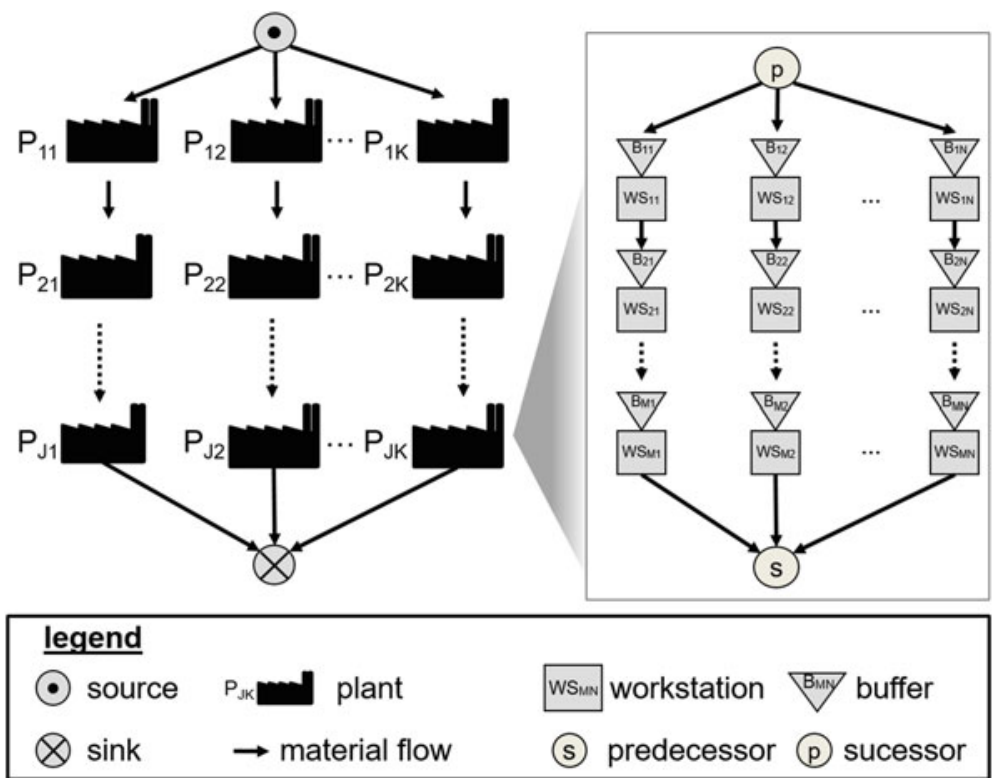

Fig. 1 Production network scenario (based on Scholz-Reiter et al. (2009d))

parallel workstations. Mainly, the scenario was applied as a $3 \times 3$ scenario, i.e., three stages and three parallel workstations per stage (Scholz-Reiter et al. 2005a, b). However, the model is scalable to any desired problem size, e.g., Scholz-Reiter et al. (2010a) applied the scenario with a maximum of $10 \times 10$ workstations.

Based on the described shop-floor scenario, a generic " $\mathrm{J} \times \mathrm{K}$ production network scenario" was developed, allowing the integration of external transportation between different production plants (see Fig. 1). Analogous to the shop-floor scenario, the network scenario consists of $\mathrm{J} \times \mathrm{K}$ production plants, i.e., each product has to be processed in one production plant on each production step. Each production plant consists of a shop-floor model with $\mathrm{N} \times \mathrm{M}$ workstations. A transport system connects the production plants. The scenario allows the definition of symmetric and asymmetric network configurations (Scholz-Reiter et al. 2009d).

Besides the scalable model size, both the shop-floor and the network reference scenarios offer various possibilities to consider different influencing factors on the methods' performance. Among others, the reference scenarios allow the variation of processing and set-up times, the consideration of different job types, fluctuation of order entrance, or disturbances like workstation breakdowns. 


\subsubsection{Transportation Scenario}

The transportation scenario covers general cargo transport (package freight) through a dynamic multi-modal transportation network. For the representation of the route network, directed graphs were used (Wenning et al. 2007a). The transportation network consists of a discrete number of geographically distributed vertices (nodes) connected by edges. A vertex (node) represents a fixed location in the transportation network, where several edges (roads) intersect, or trans-shipment is carried out or both. Vertices can be either active or passive. Active vertices include transshipment facilities, where transport units are loaded and unloaded. Passive vertices have no trans-shipment facilities. At vertices, sources are located, i.e., departure points of packages that start their journey through the transportation network. Sinks, or destinations, where packages finish their journey through the transportation network, are located at vertices as well. Edges are connections between neighboring vertices, representing, e.g., roads. They are considered to be directed and of a fixed length. A route is a connection between two different vertices that may include one or several edges. Often several different routes are possible to connect two nodes.

Packages represent pieces of goods or cargo that need to be transported from a given source to a given sink in the transportation network. A package is indivisible, which means that it cannot be broken down into smaller parts. Packages cannot move through the transportation network themselves and thus need transport units to move them between nodes of the network. A package can be loaded onto, or unloaded from, a transport unit at active vertices, allowing the exchange of transport units. It can also wait at the vertex for transport in the future. A transport order contains all information needed for carrying out the transport of a package or a group of packages. Transport units are generalized representations of transport units, e.g., trucks or vans. Transport units move between different vertices at a certain speed using the connecting edges. Different types of transport units can be restricted to different subsets of the vertices. Transport units can each take a number of cargo packages with them, which is limited by their specific capacity. At active vertices with trans-shipment capacity, some of the cargo packages can be exchanged.

The transport scenario may include dynamic and stochastic variations: New packages with transport orders are generated at run time, while packages delivered to their destination disappear. Vertices may newly appear or disappear, while edges may be temporarily unavailable for use. The speed of transport units traveling between vertices may be stochastically distributed.

Several instances of this general scenario were implemented, including a small scenario of four vertices and a so-called Germany scenario, where the location of the nodes is based on a network of 18 cities in Germany, while the edges between the vertices represented highway connections between those cities (see Fig. 2). Also, larger transportation networks of up to 87 vertices have been derived from a map of Central Europe (Wenning et al. 2007b; Wenning 2010). 


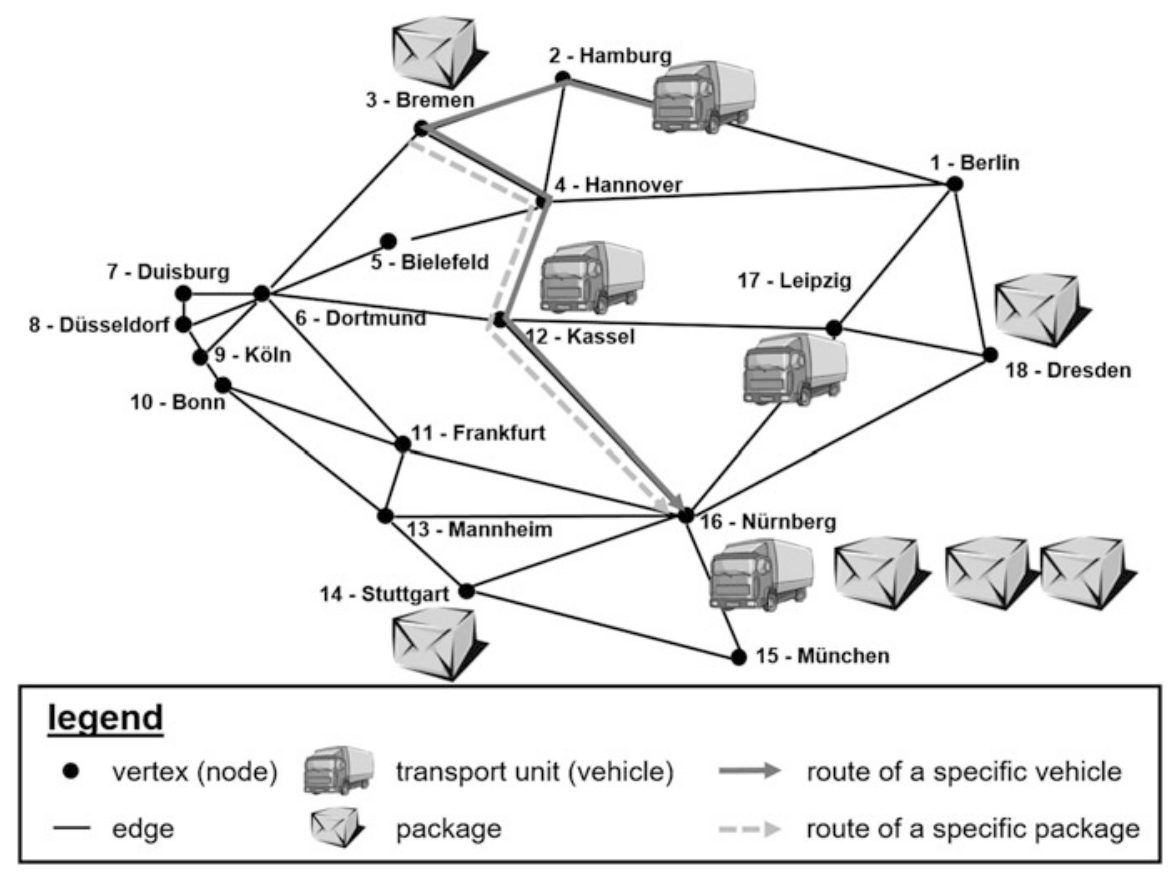

Fig. 2 Transportation scenario (based on Wenning et al. (2007b))

\subsection{Modeling Approaches}

For the modeling of autonomous control methods, mainly discrete-event simulation using the software tool eM-Plant (today known as Tecnomatix Plant Simulation) has been applied. Both discrete-event simulation and the software Plant Simulation are well established and often used for the simulation of material flow processes in manufacturing and logistics. The discrete-event modeling approach was also compared to continuous modeling using the also established System Dynamics software tool VENSIM. The comparison showed that discrete-event simulation allows a detailed description of the shop floor processes but requires high programming effort to implement the autonomous control strategies. These strategies can be implemented in continuous models rather quickly, but the shop floor processes are described only on a rather high aggregation level (Scholz-Reiter et al. 2005b).

A combination of both discrete-event and continuous approaches has been applied to analyze the stability of autonomously controlled production systems. Roughly speaking, a production system is stable if its state remains bounded over time. Therefore, a mathematical investigation using differential equations was first applied to calculate the parameters for which stability of the production system can be guaranteed. Second, the results of the mathematical stability analyses are used as a starting point for discrete-event simulation. With an enlarged set of parameters, the 
discrete-event simulation can be used to refine the calculated stability results. This combined approach takes advantage of both continuous and discrete-event modeling approaches. The main advantage lies in the high time efficiency. Since the discreteevent model uses the results of the mathematical approach as input, the detailed stability parameters can be determined in less time compared to a pure trial-anderror simulation study (Scholz-Reiter et al. 2011d).

In addition to using established software tools, the scientists within the CRC 637 also developed modeling tools that support the modeling of autonomously controlled processes in particular. A multi-agent simulation platform, the Platform for Simulations with Multiple Agents (PlaSMA), allows simulating mutual negotiations of software agents, which can represent autonomous objects (Warden et al. 2010). Also, a simulation environment for the modeling of autonomously controlled transportation processes was developed. The simulation environment uses a $\mathrm{C}++$ class library for the discrete-event simulation of communication networks (Becker et al. 2006). With the Autonomous Logistics Engineering Methodology (ALEM), a framework for the modeling of autonomous logistic processes and their infrastructure was developed (Scholz-Reiter et al. 2007b).

\subsection{Evaluation of Autonomously Controlled Systems}

The profound evaluation of the performance of autonomous control methods requires a target system that contains performance measurements for the basic categories of logistic objectives: due date adherence, throughput time, work in process, and utilization (Nyhuis and Wiendahl 2009). Therefore, Philipp et al. (2007) introduced a vectorial approach that considers these basic objectives with weighting factors to adjust the influence of each objective onto the total logistics target achievement. Grundstein et al. (2015b) enhanced the vectorial approach to additionally consider measurements of planning adherence.

The analysis of the assumed influences on the autonomous control methods' performance (cf. Sect. 2) requires measuring the scenarios' autonomy and complexity. In this context, Böse (2012) introduced a morphological pattern to specify a production system's level of autonomy. The measurement contains criteria within the categories "decision-making," "information processing," and "decision execution" and, thus, covers the fundamental characteristics of autonomous control. Grundstein et al. (2015b) expanded the morphological pattern to consider both the planning and the control level. In detail, the expansion focused on criteria to describe coupling strategies used for a combined application of conventional planning and autonomous control (see Sect. 4.3).

The measurement of the production systems' complexity considers various dimensions of complexity (Windt et al. 2008). A complexity cube describes the system's complexity within the dimensions "organizational complexity," "timerelated complexity," and "systemic complexity." Each area of the complexity cube 
can be described by a complexity vector, resulting in a complexity value for the considered production system.

The described approaches for the performance measurement and the specification of the levels of autonomy and complexity have been integrated into a so-called three-component-evaluation system (Philipp 2014; Windt et al. 2010c). The authors also stated detailed assumptions regarding the interdependencies between the dimensions of the evaluation system.

\section{Methods for Autonomous Control}

This section introduces the methods developed for autonomously controlled production systems and transportation networks. This includes, first, a method for the modeling of logistic processes and their required infrastructure components. In addition, methods for decision-making for both production and transportation and the coupling of autonomous control with conventional planning methods are introduced.

\subsection{Engineering Methodology for Autonomous Logistic Processes}

For the realization of autonomously controlled systems, the logistic objects and their required infrastructure components have to be modeled. These components depend on the selected control strategy and the architecture of the control system. In this context, the Autonomous Logistics Engineering Methodology (ALEM) offers a procedure model for configuring autonomous logistic processes and their infrastructure. The process mapping is based on a view concept that distinguishes between five views of modeling (Scholz-Reiter et al. 2007b). The "structure view" contains the representation of the logistic objects as well as their interrelationships. The required knowledge of the logistic objects for decision-making is described in the "knowledge view." The "capability view" concretizes the capabilities of the individual logistic objects. The "process view" is used to model the logical and temporal sequence of activities and states of the logistic objects. Finally, the "communication view" focuses on the content and temporal sequence of the information exchange between the logistic objects (Scholz-Reiter et al. 2007b).

The notation within the described views is based on the Unified Modeling Language (UML). UML includes various diagram types, which can be divided into structure diagrams and behavior diagrams. The static structure is represented in ALEM within the structure, the knowledge, and the capability view. Within these views, class diagrams and object diagrams are used. The description of dynamic processes in ALEM comprises the process and the communication view. 
In the process view, activity diagrams are used to describe the process flows. The communication view uses sequence diagrams to specify the temporal and logical sequence of communication between different objects (Scholz-Reiter et al. 2007b).

In addition to the described view concept and the UML-based notation, ALEM provides a procedure model to support the user during modeling. In summary, ALEM offers a set of methods and tools that have been combined within a software tool to support the mapping of autonomous logistic processes (Sowade et al. 2012).

\subsection{Autonomous Control Methods}

This section introduces different methods for autonomous decision-making in production systems and logistics networks. An overview of the developed methods is given in Table 1. Generally, the methods can be categorized according to the reference scenarios (cf. Sect. 3.1) in methods for the scheduling of shop-floor, transportation, and network processes (introduced in Sect. 4.2.2).

Besides the application area, the methods also differ in their characteristics. A common differentiation criterion is the information basis used for decisionmaking. Whereas some methods base their decision on future data (e.g., estimated processing times), others decide based on past data (e.g., recorded processing times of preceding production orders). Also, it can be differentiated between local information and information discovery methods depending on the number of planning steps considered for decision-making. A comprehensive overview of possible criteria to classify autonomous control methods is given by Windt et al. (2010a).

\subsubsection{Production Scheduling}

The queue length estimator (QLE) chooses the workstation based on the estimated waiting time, taking into account the planned processing time of each production order in the input buffer (Scholz-Reiter et al. 2008a, b). Originally, the QLE method for workstation assignment is combined with a first in first out (FIFO) control strategy, which means that already waiting production orders in the buffer are sorted according to their arrival time. A variation called due date method (DUE) is the combination of QLE with sorting the waiting orders according to their due date (Scholz-Reiter et al. 2009c). The bio-inspired pheromone method (PHE) mimics the behavior of ants when foraging. Ants find the shortest way to food by leaving pheromone trails in the environment. On the shortest and thus most frequented path, the pheromone trail is renewed most often and is most pronounced after some time. The ants follow the trail with the highest pheromone concentration. The described principle is transferred to production control as follows: Every part leaves data about the duration of the waiting and processing time when leaving a workstation. Subsequent parts make their decision based on the data left by similar products. 


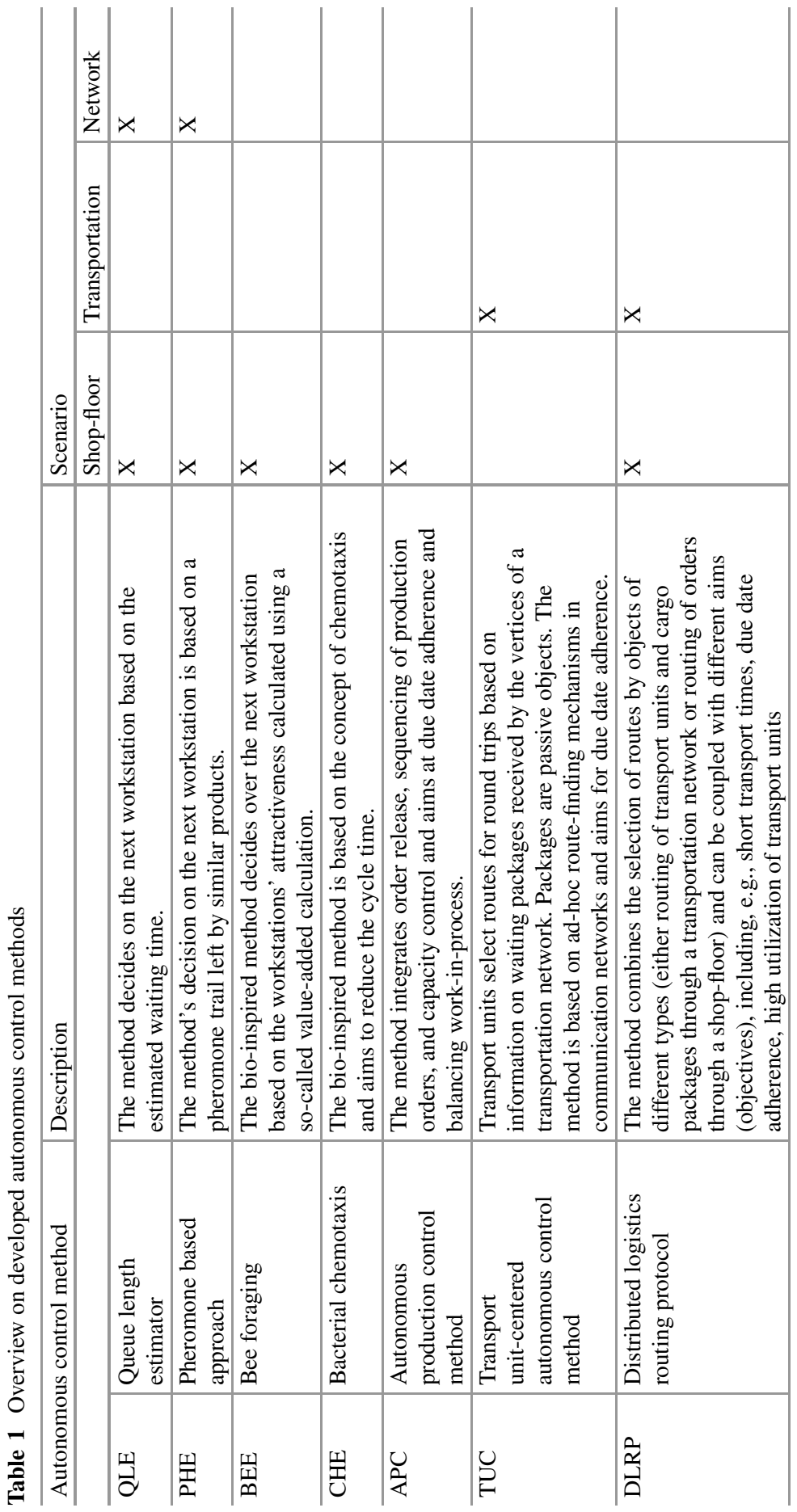


The frequency of updating or the number of data records taken into account mimics the evaporation of pheromones in ants (Armbruster et al. 2006; Scholz-Reiter et al. 2008b).

Both the QLE and the PHE methods have been applied to shop-floor scenarios with and without set-up times. Since the order sequence of waiting parts is not communicated by the pheromones, for the PHE method, a correction was used to integrate set-up times into the decision-making. Also, a weighted combination of the QLE and the PHE method, including the mentioned correction term, was developed (Scholz-Reiter et al. 2007a). The QLE and the PHE method are originally developed for production scheduling. However, both methods have been adapted for the assignment of production orders to plants in the production network scenario. The QLE method for plant assignment $\left(\mathrm{QLE}_{\mathrm{n}}\right)$ decides over the next plant, based on information about the duration of transport, waiting times, and processing times in the subsequent plants. Analogously, the PHE method $\left(\mathrm{PHE}_{\mathrm{n}}\right)$ for plant assignment decides based on past data about the necessary time to pass the transport system and the corresponding plant (Scholz-Reiter et al. 2009d).

The bee foraging approach (BEE) mimics the behavior of bees in the form of a cost-based approach. For this purpose, the attractiveness of a station for an order is calculated using a "value-added" calculation. The expected lead time and the cost rate of the station are included in this calculation (Scholz-Reiter et al. 2008b). The method of bacterial chemotaxis (CHE) is a bio-inspired method based on the concept of chemotaxis. The method aims to reduce the cycle time (Scholz-Reiter et al. 2010c).

So far, the presented autonomous control methods for shop-floor scheduling focus on workstation assignment combined with certain priority rules for order sequencing (mostly FIFO). In the simulation studies presented below, orders are mostly released to specific times that are set to a sine function. Generally, the methods could be combined with any order release method, but the influence of different order release methods on the autonomous control methods' performance was not investigated. The due date-oriented autonomous production control method (APC) closes this gap by integrating order release, sequencing of production orders, and capacity control, and thus, all production control tasks. The APC method aims to meet due dates and balancing the work-in-process on a defined level. The method requires basic planning data, e.g., processing start and completion times. Production orders are released if the planned release date is reached or if the range on a workstation on the first stage falls below a defined limit. Workstation assignment is based on due dates and throughput time to balance the workload between parallel workstations. Capacity control is triggered if the defined range limits are violated. The sequencing of production orders takes into account due dates and set-up times. The method's decisions are based on both future and past information (Grundstein et al. 2017; Grundstein 2017).

Another autonomous control method for production scheduling is the distributed logistics routing protocol for production $\left(\right.$ DLRP $\left._{\mathrm{p}}\right)$. The DLRP was originally developed for the autonomous routing of both vehicles and packages in transportation networks (see Sect. 4.2.2 below). However, the basic principles are independent of 
the original application scope and also hold advantages for production scheduling. Consequently, the DLRP was transferred to production scheduling with the basic idea that production orders should route themselves through a production system that consists of several workstations used for different consecutive operations (Rekersbrink et al. 2010).

\subsubsection{Transportation Scheduling}

The developed autonomous control methods for transportation scheduling are based on source routing mechanisms used for mobile ad-hoc networks like Wireless Local Area Network (W-LAN or Wi-Fi). Route discovery mechanisms for ad-hoc networks can be divided into proactive, reactive, and hybrid procedures (Perkins 2001). In proactive procedures, each node maintains a table with routes to the other nodes in the network. The nodes exchange appropriate messages about their current state to keep these tables updated. Reactive methods, like dynamic source routing (DSR), determine routes only when needed. The node that wants to communicate sends a route request message to its neighboring nodes. The neighboring nodes forward this route request until the target node is found. The target node responds with a route reply message to the initiator of the routing process, which is propagated back through the network. Hybrid procedures combine mechanisms of proactive and reactive ones. For example, a neighborhood zone can be defined, where most communication is expected to take place and routing is proactive, whereas routes to nodes outside the zone are determined reactively.

Wenning et al. (2005, 2006, 2007b) developed a pure transport unit-centered autonomous control method. Based on the method, each individual vehicle can make its autonomous decisions based on its specific objectives, choosing both its route and the packages to be taken. Vehicles planning their next movements use route request mechanisms to determine a round trip route to the destination vertices of the packages present at a source vertex. The packages are passive objects without intelligence. A genetic algorithm is used to optimize a vehicle target function, which includes transport costs and rewards for the transportation of packages close to their ordered delivery dates.

The distributed logistics routing protocol for transports $\left(\mathrm{DLRP}_{\mathrm{t}}\right)$ is a combined vehicle-centric and unit-goods-centric approach, which considers both transport units and packages as autonomous, intelligent objects and determines interdependent routes for both types of objects. It combines hybrid routing mechanisms for ad-hoc networks (e.g., DSR), which do not need to know the network topology, with a reactive planning component for the rescheduling of logistic objects (Rekersbrink et al. 2009; Scholz-Reiter et al. 2006a, 2009a).

Both transport units and packages present at a vertex register their destinations at that vertex. The vertex sends route requests for both types of objects to determine possible routes to their respective destinations. The route request and reply mechanism assume that a vertex only has information about itself and its direct neighboring nodes. After receiving several route proposals, the objects can 
decide on a route and register it with the involved vertices. Different route decision functions can be realized with the $\mathrm{DLRP}_{t}$. The information required for the decisions must be specified precisely in the RouteRequest and RouteReply messages so that each object can find the necessary information (Scholz-Reiter et al. 2006a). A method based on fuzzy logic is used to evaluate the different proposed routes (Rekersbrink et al. 2007).

Wenning et al. $(2010,2011)$ generalized the DLRP $_{t}$ to a more abstract autonomous control method that considers weighted combinations of different decision criteria, such as monetary costs caused by individual logistic objects, ecological costs in the form of carbon dioxide emissions, and risks (e.g., the risk of delays).

In many real-life transport processes, organizational information constraints often restrict the sharing of necessary data. To take these into consideration, Scholz-Reiter et al. (2010b) defined different actors (suppliers and transporters) and their tasks in the logistics network, including the required minimum information. To improve the scalability of information processing in large networks, various mechanisms to limit the propagation of transport requests can be added to the $\operatorname{DLRP}_{\mathrm{t}}$. These include limitation of the search depth, intermediary assessment of partial routes for segments of the total route, re-using data from previous request propagations (Wenning et al. 2009; Wenning 2010), or mechanisms for clustering and regional partitioning of transportation networks (Singh et al. 2008).

\subsection{Coupling of Conventional Planning and Autonomous Control}

The concept of coupling aims at combining the high efficiency of autonomous control in dynamic production environments with the high planning accuracy of conventional planning methods in static production environments. Thus, conventional planning and autonomous control methods must be enabled to exchange decision-relevant information. This information exchange allows the consideration and execution of necessary adaptions due to occurring dynamic influences and resulting deviations of predefined planning results. Coupling strategies define the extent and the duration of coupling. The extent specifies to which degree autonomous control refers to central planning parameters for decision-making (e.g., predefined workstation assignments or sequences of production orders). The duration defines if coupling is applied permanently or depending on the system state. In the latter case, production orders usually follow the predefined schedule, and autonomous control is only applied if deviations of the schedule are expected. The described coupling strategies have been applied to couple different conventional planning heuristics with several autonomous control methods for production scheduling (Schukraft et al. 2016a). 


\section{Evaluation of Autonomous Control}

The autonomous control methods presented above have been evaluated in various simulation studies using either discrete-event or continuous modeling approaches for the shop-floor scenario. For the transportation scenario, also multi-agent system simulations were used. The simulation results presented in the following are mainly based on the introduced reference scenarios for production systems and transportation networks (cf. Sect. 3.1). For production scheduling, the shop-floor scenario was mainly applied as a $3 \times 3$-model, but also larger problem sizes up to $10 \times 10$ workstations were used. Most studies considered various job types, heterogeneous processing times, and sequence-dependent set-up times. Mostly, order entry was modeled using a certain arrival rate set to a sine function. This also allowed a variation of the dynamic influence by adjusting period and amplitude of the sine function. The simulation studies performed to assess the autonomously controlled transportation methods used transportation networks with 18,40 , or 87 vertices. Different criteria were used as performance indicators. These included aggregate transportation times and distances in relation to the shortest paths, delivery time adherence of packages, utilization of transport units, and numbers of queued packages.

\subsection{Performance of Autonomous Control and Influence of Complexity}

The performance of autonomous control methods has been compared in different simulation studies. Several studies focused on the QLE and the PHE algorithm, which differ in their information basis used for decision-making (future vs. past data). Other studies compared the QLE method with the $\mathrm{DLRP}_{\mathrm{p}}$ to compare local information and information discovery methods.

The QLE outperforms the PHE method in high dynamic environments in both the shop-floor scenario (Scholz-Reiter et al. 2006b, 2008b) and the network scenario (Scholz-Reiter et al. 2011b). The PHE performs best in situations with fewer dynamics in the arrival rate. This could be explained since the PHE method's decision-making is based on past data, and thus, the method needs time to react to changing conditions in dynamic environments. Also, a weighted combination of both methods for decision-making leads to a promising performance (Scholz-Reiter et al. 2007a; Scholz-Reiter and Jagalski 2008).

The application of the QLE and the $\operatorname{DLRP}_{\mathrm{p}}$ in a shop-floor scenario with up to $10 \times 10$ lines and workstations showed that both methods reach a comparable performance with slightly better values for the QLE method. It was assumed that the DLRP is designed for more complex environments, and obviously, the simpler decisions of the QLE are more efficient for the used shop-floor scenario (ScholzReiter et al. 2010a, 2011c). 
Some studies also analyzed the impact of different complexity levels on the methods' performance. Scholz-Reiter et al. (2006c) applied the QLE and the PHE method in a shop-floor scenario. Several complexity levels were considered by using different network sizes and a different number of job types. The QLE method is not affected by a larger system size since the throughput time was always close to the minimal throughput time (calculated with minimal processing time for each production stage). Contrarily, the performance of the PHE algorithm gets worse with increasing system size. For an increasing number of product types, the QLE also outperforms the PHE method. However, the performance of the PHE gets better with a rising number of product types.

Grundstein et al. (2015a) analyzed the impact of different order release methods on the performance of autonomous control. The study applied the order release methods immediate order release (IMR), due date oriented (DATE), and constant work-in-process (CONWIP) in combination with the autonomous control methods QLE, QLE with sorting the production according to the due date (DUE), and PHE. The method combinations were applied in a shop-floor scenario with different levels of dynamics for order arrival. The results show no dominant order release method. Instead, the performance depends on the dynamic level for order arrival and the pursued logistic objective.

The APC method was applied in a shop-floor scenario. The complexity was varied by using different types and intensities of dynamic influences. The method's performance was, among others, compared with the QLE method and conventional planning for the task of workstation assignment. The results show that the performance for all method combinations decreases with an increase of dynamic influences. The application of APC for all control tasks outperforms other method combinations for customer-oriented target profiles with a high weighting of due date-oriented objectives. For other profiles that favor low work-in-process, a combination with the QLE method for workstation assignment reaches higher values. This is explained by the fact that the APC method consciously aims at high due date adherence at the cost of high work-in-process (Grundstein 2017). The results also confirmed the findings of a previous simulation study that used a similar scenario but fewer method combinations and dynamic influences (Grundstein et al. 2015c).

The influence of autonomy and complexity on the performance of autonomous control methods has also been analyzed by using real production data from a power plant supplier. The production system consists of several work systems, each with a different number of workstations. The levels of complexity and autonomy have been defined using the three-component evaluation system described in Sect. 3.3. Different studies focused on different aspects to define the levels of complexity and autonomy. Philipp (2014) and Windt et al. (2010c) addressed complexity by varying the number and diversity of workstations, orders, material flow connections, and manufacturing processes. The level of autonomy varied depending on the applied planning and control logic. Scholz-Reiter et al. (2009c) kept the shop-floor scenario constant but used machine availability as a parameter to vary the level of complexity. The autonomy was varied by the number of work systems controlled by autonomous control methods. Philipp (2014) and Windt et al. (2010c) showed that the logistics 
performance improves with an increasing level of autonomy and decreases again with the highest autonomy level. The decreasing performance may lie in a chaotic behavior of the logistic objects, which is not in the sense of the overall goal when the degree of autonomy is too high. Scholz-Reiter et al. (2009c) also showed that for each complexity level, an increase of autonomy improved the logistic performance. This effect is even enhanced with rising complexity levels. Contrary to Philipp (2014) and Windt et al. (2010c), a decrease for the highest level of autonomy could not be observed. The different behavior of autonomous control in combination with a high autonomy level can probably be explained by the authors' different approaches for the definition of autonomy and complexity.

For transportation scheduling, various complexity levels for the vehicle-centric approach were compared (Wenning et al. 2006). It was shown that selecting routes solely based on packages locally available at a vehicle's starting point led to low utilization of vehicle capacities. The inclusion of packages, which can be loaded en route, significantly improved vehicle utilization and resulted in fewer vehicles needed for transport. The possibility of re-planning at each node, i.e., discarding the previous route and re-optimizing it on the basis of current data, was also investigated. The results showed that frequent rescheduling does actually impair the results compared to one-off route planning with consideration of the general cargo at other nodes. This can be explained by the fact that optimization was carried out for a complete route, of which only the first section was actually traveled before the rescheduling begins. This loss of performance could be partially compensated by a higher weighting of local general packages. Nevertheless, frequent rescheduling of routes can be disadvantageous.

For the $\operatorname{DLRP}_{t}$, it could be shown that measures to improve the scalability of information processing can significantly reduce communication volumes in large networks without impairing the logistical achievement of objectives. For that reason, the $\mathrm{DLRP}_{\mathrm{t}}$ can be scaled to larger networks within the given limits of the communication infrastructure (Wenning et al. 2009).

So far, the results show that the autonomous control methods are suitable for production, transportation, and network scheduling and able to cope with complex environments and dynamic influences. Also, the results show that the methods' performance depends on the complexity level of the scenario. Furthermore, it was observed that the methods' performance depends on its characteristics (e.g., futureor past-oriented autonomous control methods).

\subsection{Autonomous Control and Conventional Planning and Control}

Autonomous control methods have been developed as an alternative to conventional planning and control methods. Thus, a profound evaluation of autonomous control requires a comparison between both autonomous control and conventional planning 
and control. This issue is addressed in different simulation studies. These studies mostly considered several scenarios with varying levels of dynamic influences. Scholz-Reiter et al. (2008a) compared conventional planning and control with the BEE algorithm in a shop-floor scenario and analyzed the impact of workstation breakdowns on the methods' performance. The results showed that the methods' performance was comparable without disturbances. In contrast, in the case of occurring workstation breakdowns, the BEE algorithm showed slightly better results. Scholz-Reiter and Jagalski (2008) used a $3 \times 3$ shop-floor scenario to compare conventional planning and control with the PHE method. The autonomous control method reached a better performance and seemed to be more robust against dynamic influences. Scholz-Reiter et al. (2009d) compared autonomous control with conventional planning and control in a production network scenario. An autonomously controlled network seemed to react more robustly to both changes in the order arrival rate and variations of the transport interval between the plants. Whereas the previously mentioned studies used rather simple methods for conventional planning and control, Scholz-Reiter et al. (2010a) applied more sophisticated methods, including several constructive heuristics and a genetic algorithm introduced by Jungwattanakit et al. (2009). The study considered different dynamic levels using a Poisson process with a varying number of released jobs per time unit. The results showed that the conventional planning methods performed best in static environments, whereas the performance of autonomous control improved with increasing dynamics. In highly dynamic environments, autonomous control outperformed conventional planning.

For transport scheduling, the DLRP $\mathrm{t}_{\mathrm{t}}$ was compared with benchmark solutions for the dynamical Vehicle Routing Problem (dynVRP) and the dynamic Pickup and Delivery Problem with Time Windows (dynPDPTW), like Tabu-Search and Mixed Integer Programming (MIP). The result showed that, for complex dynamical Vehicle Routing Problems with large problem sizes, the $\mathrm{DLRP}_{\mathrm{t}}$ outperforms classical solutions (Rekersbrink et al. 2009; Scholz-Reiter et al. 2009a). Regarding the dynPDPTW problem, the performance achieved by the DLRP ${ }_{t}$ was comparable, and in some cases superior, to the benchmark solutions. In addition, compared to classical optimization approaches, the $\mathrm{DLRP}_{t}$ offers extended degrees of freedom and variability, e.g., object individual decision functions (Rekersbrink and Wenning 2011).

Schukraft et al. (2015) applied different coupling strategies for the combined application of conventional planning and autonomous control methods in a shopfloor scenario similar to Scholz-Reiter et al. (2010a). Depending on the extent of autonomous control for decision-making, these strategies can be rated from a low to a high level of autonomy. The performance measurement considered both logistic objectives achievement and adherence to predefined planning parameters. For production planning, several constructive heuristics have been used, introduced by Jungwattanakit et al. (2009). For autonomous control, the QLE and the PHE algorithms in combination with different priority rules have been applied. The simulation study considered different levels of dynamic influences to varying the complexity of the shop-floor scenario. The results show that there is no dominant coupling 
strategy for all scenarios and performance measurements. Naturally, high adherence to planning parameters supports a high planning accuracy. Especially strategies with autonomous order sequencing reach high logistic objectives achievement. In high dynamic environments, coupling strategies with a higher level of autonomy mostly outperform those with less autonomy. In contrast, in static environments, strategies with high adherence to preplanned parameters reach better results. These results could also be confirmed in a similar simulation study based on real data (Schukraft et al. 2016b).

Generally, the comparisons of autonomous control and conventional planning and control methods confirm the initial hypothesis that autonomous control methods are especially promising in high dynamic environments. In contrast, conventional planning and control methods reach comparable or even better results in static environments. Although some of the results are based on very simple conventional planning and control methods, the results could also be confirmed with more sophisticated conventional planning methods. Furthermore, the coupling of conventional planning and autonomous control showed that the performance also depends on the level of autonomy.

\section{Application of Autonomous Control}

So far, the presented results were mostly based on the theoretical reference scenarios described in Sect. 3.1. These scenarios are well suitable for the development and evaluation of autonomous control methods. However, they do not fully consider influencing factors that occur in practical application. Thus, to evaluate and prove the applicability of autonomous control in practice, the methods have also been applied in several real-world use cases described in the following.

\subsection{Finished Vehicle Logistics}

Finished vehicle distribution logistics covers all distribution processes of readymade cars (and other vehicles) from the original equipment manufacturers (OEM) to the end customers, involving the OEM, various logistic service providers (LSP), and car dealers (Scholz-Reiter et al. 2012). It is an important branch within logistics, as many millions of cars have to be transported each year, a large part of them across countries and continents. The so-called vehicle compounds situated at focal points within the finished vehicle distribution networks, e.g., seaports, serve as transshipment and service points. At large compounds, several million vehicles per year can change the means of transport (trucks, railways, and ships). In addition, vehicles may be stored there intermediately for weeks or months, and technical operations may be performed, including washing, removing protective coats, installing luxury equipment, or technical modifications. At any given time, there can be up to 100,000 
vehicles on a large compound (Klug 2018). For each of these vehicles, arrival (unloading), parking (storage), technical service operations, and finally departure (loading) have to be planned and executed. Thus, vehicle compound operators face difficult, complex, and highly dynamic planning and control tasks, rendering them a worthwhile application for autonomous control.

Consequently, a mechanism for parking space allocation was developed, which considers both the parking vehicles and the parking spaces as fully autonomous objects. Both object types can negotiate with each other and render their own decisions based on their local sets of objectives. The goal of the parking spaces is the highest possible occupation, and thus, unoccupied parking spaces will offer parking services for requesting vehicles. The objectives of the vehicles are to minimize their overall travel times on the compound. Therefore, a vehicle will choose the parking space that offers the shortest possible travel time from the unloading point to the parking space and from there to the subsequent destination (either a service station or the allocation area to the subsequent transport units). A negotiation mechanism regulates the interaction between the vehicles and the parking areas as follows:

1. Request of the vehicle for parking space, including its starting location and its destination.

2. Computation of distances and resulting travel times to an unoccupied parking area, resulting in an offer by that parking area that includes the travel time.

3. Comparison of all offers from different unoccupied parking areas by the requesting vehicle and selection of the parking area with the minimum travel time.

This negotiation mechanism was implemented using a multi-agent system. A simulation study was applied to compare the mechanism's performance to a central parking assignment based on fixed priority rules. The simulation study resulted in a reduced average vehicle travel time, equating to a time saving of 112 person workdays over a year for the studied compound (Böse et al. 2009; Böse and Windt 2007a). The improvements were caused by the capability to select the best available out of several alternative parking area offers.

Later simulation studies extended the scope of the autonomously controlled scenario by adding optional passage of the vehicles through a technical service center. In addition, different methods from autonomous control and related fields (e.g., DLRP $_{t}$, pheromone Holonic, minimum buffer, QLE) were compared to conventional methods, e.g., predefined lists, corresponding to how cars were actually distributed on the studied compound. The simulations compared average travel times to parking and average parking area utilization for the storage processes. For the manufacturing processes, average total operation times of the vehicles and average due date reliability have been used. The results showed that the autonomous control methods could outperform the conventional methods under certain conditions, but no method dominates all others uniformly. Consequently, different areas of application exist for specific methods. In addition, preferences for different logistic objectives influence the selection of an appropriate autonomous control method. For instance, methods aiming at minimization of traveling times 
may cause low utilization of the parking areas. In contrast, other methods may cause opposite effects (Becker and Windt 2011; Windt et al. 2010b).

\subsection{Apparel Logistics}

As another application scenario, apparel distribution logistics was studied. Apparel logistics face conflicting demands of short delivery times, high product, and variant diversity, as well as uncertain delivery times due to the spatial separation of manufacturing in developing countries and customers in industrialized countries. For short-lived fashion products, seasonal order and delivery cycles are adopted, where many small-sized orders by individual retailers are aggregated into a large production lot that is delivered several times a year. Due to the long delivery times, which can extend to several months, some customer orders have to be anticipated using forecasts, while some orders that were initially placed are retracted or become obsolete. Consequently, some re-allocation of ready-made goods between different customer orders is necessary during distribution (Ait-Alla et al. 2014). Standardized routing causes bottlenecks in the distribution centers, including short-term peaks of stocks and workload.

In this regard, a mechanism for dynamic allocation of bundles of clothing articles, which are located at different locations within the distribution network, to customer orders was developed. Within each bundle, the individual garments could be identified using radiofrequency identification (RFID), and the composition of the bundle be compared to that of a customer order. Thus, restrictions regarding the ordered article variants (like different sizes and colors), quantities, and delivery dates can be taken into account (Scholz-Reiter et al. 2011a). The individual identification of goods and the allocation of goods packages to orders were implemented in a prototypical hardware and software application. The developed procedure was assessed by process simulation with regard to the achievable process potentials and by an economic efficiency analysis in terms of its economic effects (Scholz-Reiter et al. 2009b). Applying autonomous control to apparel logistics, however, proved to be difficult. In particular, a large number of products, each of limited value, limited technologies that could be attached (Scholz-Reiter et al. 2011a).

\subsection{Event Logistics}

Another application scenario was event management. Event management is concerned with the temporary renting of diverse equipment for use during organized events (e.g., public music concerts), its timely provision at the outside event venues, and setup in due time before the start of the event, as well as its dismantling and removal after the end of the event. Logistic processes associated with event management include the commissioning and transportation of highly diverse event 
equipment between venues, with strict deadline restrictions. They are characterized by a high level of dynamics and complexity (Harjes and Scholz-Reiter 2013b). In particular, order scheduling, which combines the allocation of resources to orders and the planning of cargo composition and transport routes for transports to, from, and between venues, seems an appropriate application area for autonomous control methods. Unexpected occurrences require frequent rescheduling of resources. The associated planning and control processes, particularly scheduling, require a correspondingly high degree of flexibility (Harjes and Scholz-Reiter 2013a). In response, an autonomous controlled scheduling system was developed. The scheduling is executed through mutual negotiations of autonomous objects represented by software agents, using the PlaSMA multi-agent simulation platform. A PlaSMA installation was set up that can transfer the current order situation of an event management company into a scenario, process simulations, and then transform the simulation results into planning decisions, generating event-specific picking and loading lists and a transport route and personnel schedules.

Within the PlaSMA installation, corresponding agents have been created to represent articles, vehicles, and employees. To start a planning cycle, the so-called list agents are created for advertised events, which coordinate the disposition of the resources for their respective events. For this purpose, they are equipped with event data like location, duration, and requested equipment. To start a negotiation, the list agent sends a request to all individual items of the types of equipment requested for the respective event. The requested item agents check the availability of their items for the event duration (they might already be scheduled for another parallel event or maintenance). Agents of available items send a request to the vehicle agents to clarify their movement to the event location. The vehicle agents, in turn, check whether, and at what cost, transport of the respective item to the destination is possible and then send corresponding offers or rejections to the items. For the generation of route plans, the $\mathrm{DLRP}_{\mathrm{t}}$ protocol was implemented into the PlaSMA environment as a behavioral scheme of the transport agents (Harjes and Scholz-Reiter 2013a). This protocol allows the transport agents to find routes in a closed transportation network that is dynamically variable due to the only temporary existence of the event locations.

In a second turn, corresponding offers or rejections are transmitted in reverse order by the item agents to the initiating list agent. The list agent then selects the best of all offers it has received from items. The negotiation is complete when a list agent has been able to allocate the requested resources to its event. When the negotiation cycles have been completed for all list agents, all events are planned, and the simulation ends. If the scheduling cannot be completed with the available resources, the project manager must allow the use of additional rental vehicles or items, which are taken into account in a new simulation run. This procedure can be continued until a solution to the scheduling problem has been found. The final allocation of resources to projects can take several iterations (Harjes and ScholzReiter 2013a).

To enable the consideration of items already located at event locations, a hardware module for the acquisition and processing of the necessary material 
flow data at the event locations was developed, using RFID technology to identify equipment articles. The module can recognize the direction of loading and transmit the data obtained to the disposition system in a processed form.

As a result, the system can reduce the workload of experts involved in central planning and increase the efficiency and robustness of the planning under dynamic conditions. The simulations showed that for the studied event management company, the developed autonomous control system could reduce the number and aggregate distance of trips by between 20,000 and 52,000 km per year (Harjes and Scholz-Reiter 2014a, b).

\subsection{Material Supply for Production}

The internal logistics within production systems often uses the Kanban method (Ōno 2013). However, a lack of coordination between order release and order execution may cause problems during peak periods with unusually high demand. An intelligent network of different logistics units can improve the coordination between release and execution of the order. Correspondingly, a concept for a cyber-physical logistics system was developed by researchers from BIBA, which uses adapted autonomous control methods to realize an efficient, demand-oriented material supply in production (dynamic milk run). The concept was implemented in cooperation with a manufacturer of gear wheels, whose intra-logistics is based on a container-Kanban-procedure in combination with a milk run. In front of every machine, there is a delivery space where a one-floor roller (transport unit for several load carriers) for precisely one production order can be put down or picked up again. An employee picks up finished orders and delivers supplies to the machines with a tugger train. The machines are arranged so that the employee can reach all machines by driving an "eight" shaped course. At the intersection of both loops, the employee can optionally turn to the area for incoming and outgoing goods. Originally, the employee picked off finished orders, distributed them, and noted empty delivery areas in a fixed hourly turn. In the following cycle, he equipped these free delivery spaces with orders from the buffer stock. Since the employee did not have any information about the current collection and delivery orders, he drove all loops, although there was no need for transport in some loops. The fixed hourly cycle times meant that machines spent some idle time before new orders arrived, and the tugger train was not always fully utilized (Thoben et al. 2014).

The development of a cyber-physical logistics system focused on improved synchronization of the milk run with the actual demand through the networking of individual logistics units. Demand-driven supply of materials needs ongoing information about the current occupancy of delivery and pickup spaces.

For communication of stock levels, load carriers were equipped with sensors and a communication module, turning them into cyber-physical load carriers. The sensors could locate the load carriers and monitor environmental conditions (e.g., temperature, acceleration) affecting the components. The communication module 
could transmit delivery and pickup space-related data to a mobile data processing unit, which was provided to the tugger train driver. It ran software using the transmitted data to determine (remaining) machine processing times and completion dates of the current production orders in process and calculate the optimal departure time for a new delivery and pickup cycle.

The described cyber-physical logistics system matches autonomous control concepts with Industry 4.0 related technologies and thus emphasizes the close connection between both concepts as stated in Sect. 2. The potential of such a cyberphysical logistics system was assessed by a simulation study. The simulation results illustrated that the use of cyber-physical systems for a demand-driven material supply leads to a reduction of driven loops and an apparent reduction of driven cycles, compared to the previous situation. As a demand-driven material supply may lead to an increase of transport orders in a cycle, increasing the capacity of the tugger train may lead to a stronger reduction of the driven loops (Lappe et al. 2014; Thoben et al. 2014).

\section{Conclusion and Outlook}

\subsection{Conclusion}

This chapter summarized the research activities of BIBA in the development of autonomously controlled production systems and logistics networks that started in 2004 with the Collaborative Research Center 637 "Autonomous Cooperating Logistic Processes: A paradigm Shift and its Limitations" (cf. Sect. 1). In this context, this chapter presented autonomous control methods that have been developed for both autonomously controlled production systems and transportation networks. The first evaluation based on theoretical reference scenarios showed that autonomous control in large parts meets the expectations placed in it. This will briefly be explained, referring to the hypotheses regarding the performance of autonomous control (see Sect. 2) and the results of the theoretical evaluation (see Sect. 5).

The autonomous control methods have been applied in shop-floor, production network, and transportation scenarios with different types and intensities of complexity and dynamics. Often considered influences have been, e.g., fluctuating order arrivals, workstation breakdowns, or different network sizes. The results showed that autonomous control is able to deal with such influences and still maintain high performance (cf. hypothesis 1). Also, it could be shown that the methods' performance depends on the levels of complexity and autonomy. Naturally, a rising complexity leads to a decreasing performance (cf. hypothesis 3). However, in most cases, autonomous control is able to outperform conventional planning and control methods in environments of high complexity and dynamics. Contrarily, conventional planning and control methods reach comparable or even better results in static environments (cf. hypothesis 2). Although some of these results are 
based on a comparison of autonomous control with very simple conventional planning and control methods, the results could also be confirmed with more sophisticated conventional planning and control methods. Furthermore, approaches for the coupling of conventional planning methods with autonomous control showed that a rising level of autonomy increases the efficiency of production planning and control. Furthermore, a coupled application of conventional and autonomous control is promising to combine high efficiency with higher planning accuracy than that offered by autonomous control without consideration of conventional planning (cf. hypothesis 4).

In addition to the simulation-based evaluation, autonomous control was also applied in several application scenarios based on real-world use cases (see Sect. 6). These applications generally confirm the theoretical results and show that autonomous control has a high potential to improve the efficiency of planning and control in practice. For some application areas, however, autonomous control methods may not offer the expected performance improvements, or, e.g., in apparel logistics, may be too expensive to implement.

To summarize, it can be said that the hypotheses stated in Sect. 2 could be proven. However, production systems and transportation networks, in reality, are influenced by several factors of complexity and dynamics that could not be completely covered in the applied scenarios. Further research needs to consider further influences of complexity on the methods' performance and analyze the interdependencies between different influencing factors. In addition, the theoretical scenarios also neglect influences that are crucial for practical application and should be addressed in further research. This includes, e.g., the consideration of the influence of internal transport for material supply and the transport of products between different shops and workstations.

\subsection{Outlook}

The ongoing development of Industry 4.0 goes along with the anticipated improvements in ICT necessary for the implementation of autonomously controlled systems. Autonomous control and Industry 4.0 are supplemental concepts (cf. Sect. 2): Industry 4.0 provides the technological basis necessary for the decision-making of autonomous logistic objects, whereas autonomous control contributes the decision logic in the form of methods and algorithms to increase efficiency in logistics systems.

Among the real-life application examples of autonomous control (cf. Sect. 6), the development of a cyber-physical logistics system for material supply is particularly suited to illustrate the synergy effects between autonomous control and Industry 4.0. In addition, some of the developed autonomous control methods use a representational form of autonomous control, where physical logistic objects are represented by a digital object within a software system. Different types of logistic objects are represented by different software agents, using a MAS infrastructure 
for interaction. Examples are the negotiation between packages and transport units within the transportation scenario (cf. Sect. 4.2.2) and the negotiation between vehicles and parking spaces in the vehicle compound application (cf. Sect. 6.1). This virtual representation of physical objects transitioned into the concept of the digital twin within Industry 4.0 (Negri et al. 2017).

Until now, the research in autonomously controlled systems in the context of Industry 4.0 at BIBA was steadily continued. For example, current research work in the field of vehicle compound operations for finished vehicle logistics focuses on a combined optimal control system for order assignment and shuttle routing. Shuttles are used on vehicle compounds to transport handling employees to their subsequent order. A control algorithm assigns driving orders to handling employees and transport orders to shuttles and coordinates the routing of the shuttle buses (Hoff-Hoffmeyer-Zlotnik et al. 2020). The technical implementation uses both satellite-based and indoor positioning systems to locate vehicles, as well as WLAN connectivity. Another application concerns the development of cyber-physical transport bins that can monitor components being delivered within automotive supply chains. The transport bins are equipped with mobile wireless sensors and, via mobile communication technologies, connected to digital services hosted in cloud computing systems (Teucke et al. 2018). While the cyber-physical transport bins cannot render decisions on their own, they do possess certain forms of intelligence or information processing capability.

The continuing development of Industry 4.0 related technologies like cyberphysical systems and the Internet of Things and their introduction into the industrial application will make logistic objects progressively more intelligent and connected and bring new opportunities to realize autonomous control in manufacturing and logistic processes.

Acknowledgments The research presented in this chapter was funded by German Research Foundation (DFG) as part of the Collaborative Research Centre 637 "Autonomous Cooperating Logistic Processes-A Paradigm Shift and its Limitations" and the research project "Methods for the interlinking of central planning and autonomous control in production," by the German Federal Ministry for Economic Affairs and Energy (BMWi) within the project "SaSch—Digital Services for Shaping Agile Supply Chains," by the German Federal Ministry of Education and Research (BMBF) within the project "Cyber Physical Production Systems-Enhancement of Productivity and Flexibility by Networking Intelligent Systems in the Factory," and by the German Federal Ministry of Transport and Digital Infrastructure (BMVI) within the project "ISABELLAAutomobile logistics in sea- and inland ports: interactive and simulation-based operation planning, dynamic and context-based control of device- and load movements."

\section{References}

Ait-Alla, A., Teucke, M., Lütjen, M., Beheshti-Kashi, S., Karimi, H.R.: Robust production planning in fashion apparel industry under demand uncertainty via conditional value at risk. Math. Probl. Eng. 2014, 1-10 (2014). https://doi.org/10.1155/2014/901861 
Alkan, B., Vera, D.A., Ahmad, M., Ahmad, B., Harrison, R.: Complexity in manufacturing systems and its measures: a literature review. Eur. J. Ind. Eng. 12, 116-150 (2018). https://doi.org/ 10.1504/EJIE.2018.089883

Armbruster, D., de Beer, C., Freitag, M., Jagalski, T., Ringhofer, C.: Autonomous control of production networks using a pheromone approach. Phys. A: Stat. Mech. Appl. 363, 104-114 (2006). https://doi.org/10.1016/j.physa.2006.01.052

Becker, T., Windt, K.: A comparative view on existing autonomous control approaches: observations from a simulation study. In: Hülsmann, M., Scholz-Reiter, B., Windt, K. (eds.) Autonomous Cooperation and Control in Logistics, pp. 275-289. Springer, Berlin, Heidelberg (2011)

Becker, M., Wenning, B.-L., Görg, C.: Integrated simulation of communication networks and logistical networks. In: Modeling and Simulation Tools for Emerging Telecommunication Networks, pp. 279-287. Springer, Boston, MA (2006)

Böse, F.: Selbststeuerung in der Fahrzeuglogistik: Modellierung und Analyse selbststeuernder logistischer Prozesse in der Auftragsabwicklung von Automobilterminals. In: Schriftenreihe: Informationstechnische Systeme und Organisation von Produktion und Logistik, vol. 14. Gito, Berlin (2012)

Böse, F., Windt, K.: Autonomously controlled storage allocation on an automobile terminal. In: Hülsmann, M., Windt, K. (eds.) Understanding Autonomous Cooperation and Control in Logistics, pp. 351-363. Springer, Berlin, Heidelberg (2007a)

Böse, F., Windt, K.: Catalogue of criteria for autonomous control in logistics. In: Hülsmann, M., Windt, K. (eds.) Understanding Autonomous Cooperation and Control in Logistics, pp. 57-72. Springer, Berlin, Heidelberg (2007b)

Böse, F., Piotrowski, J., Scholz-Reiter, B.: Autonomously controlled storage management in vehicle logistics - applications of RFID and mobile computing systems. Int. J. RF Technol. 1, 57-76 (2009). https://doi.org/10.1080/17545730802294452

Bouhai, Grayson, S.: Internet of Things - Evolutions and Innovations, vol. 4. John Wiley \& Sons, Hoboken, NJ (2017)

Broy, M.: Cyber-Physical Systems: Innovation Durch Softwareintensive Eingebettete Systeme. Springer, Dordrecht (2010)

Chaouchi, H.: The Internet of Things: Connecting Objects to the Web. Wiley, Somerset (2013)

Duffie, N., Bendul, J., Knollmann, M.: An analytical approach to improving due-date and lead-time dynamics in production systems. J. Manuf. Syst. 45, 273-285 (2017). https://doi.org/10.1016/ j.jmsy.2017.10.001

Freitag, M., Herzog, O., Scholz-Reiter, B.: Selbststeuerung logistischer Prozesse - Ein Paradigmenwechsel und seine Grenzen. Industrie Management. 20, 23-27 (2004)

Grundstein, S.: Fertigungsregelung flexibler Fließfertigungen und Werkstattfertigungen zur Einhaltung von Lieferterminen. GRIN Verlag, Munich (2017)

Grundstein, S., Schukraft, S., Scholz-Reiter, B., Freitag, M.: Coupling order release methods with autonomous control methods - an assessment of potentials by literature review and discrete event simulation. Int. J. Prod. Manag. Eng. 3, 43-56 (2015a)

Grundstein, S., Schukraft, S., Scholz-Reiter, B., Freitag, M.: Evaluation system for autonomous control methods in coupled planning and control systems. Procedia CIRP. 33, 121-126 (2015b). https://doi.org/10.1016/j.procir.2015.06.023

Grundstein, S., Schukraft, S., Freitag, M., Scholz-Reiter, B.: Planorientierte autonome Fertigungssteuerung: Simulationsbasierte Untersuchung der Planeinhaltung und der logistischen Zielerreichung. wt Werkstattstechnik online. 105, 220-224 (2015c)

Grundstein, S., Freitag, M., Scholz-Reiter, B.: A new method for autonomous control of complex job shops - integrating order release, sequencing and capacity control to meet due dates. J. Manuf. Syst. 42, 11-28 (2017). https://doi.org/10.1016/j.jmsy.2016.10.006

Harjes, F., Scholz-Reiter, B.: Agent-based disposition in event logistics. Res. Prod. Logist. 3, 137 150 (2013a)

Harjes, F., Scholz-Reiter, B.: Selbststeuerndes Routing für Verleihartikel. Industrie Management. 29, 44-48 (2013b) 
Harjes, F., Scholz-Reiter, B.: Autonomous control in closed dynamic logistic systems. Procedia Technol. 15, 313-322 (2014a). https://doi.org/10.1016/j.protcy.2014.09.085

Harjes, F., Scholz-Reiter, B.: Integration aspects of autonomous control in event logistics. Res. Logist. Prod. 4, 5-20 (2014b)

Hermann, M., Pentek, T., Otto, B.: Design principles for Industrie 4.0 scenarios. In: 49th Hawaii International Conference on System Sciences, pp. 3928-3937 (2016)

Hoff-Hoffmeyer-Zlotnik, M., Sprodowski, T., Freitag, M.: Revisiting order assignment problems in a real-case vehicle compound scenario. In: 2020 6th IEEE Congress on Information Science and Technology, pp. 395-400. IEEE, Piscataway (2020)

Hon, K.K.B.: Performance and evaluation of manufacturing systems. CIRP Ann. 54, 139-154 (2005). https://doi.org/10.1016/S0007-8506(07)60023-7

Jungwattanakit, J., Reodecha, M., Chaovalitwongse, P., Werner, F.: A comparison of scheduling algorithms for flexible flow shop problems with unrelated parallel machines, set-up times, and dual criteria. Comput. Oper. Res. 36, 358-378 (2009). https://doi.org/10.1016/ j.cor.2007.10.004

Kagermann, H, Wahlster, W., Helbig, J.: Umsetzungsempfehlung für das Zukunftsprojekt Industrie 4.0. Abschlussbericht des Arbeitskreises Industrie 4.0. aca-tech - Deutsche Akademie der Technikwissenschaften e. V. (2013)

Klug, F.: Logistikmanagement in der Automobilindustrie. Springer, Berlin, Heidelberg (2018)

Lappe, D., Veigt, M., Franke, M., Kolberg, D., Schlick, J., Stephan, P., Guth, P., Zimmerling, R.: Vernetzte Steuerung einer schlanken Intralogistik: Simulationsbasierte Potentialanalyse einer bedarfsorientierten Materialversorgung in der Fertigung. wt Werkstattstechnik online. 3, 112 117 (2014)

Negri, E., Fumagalli, L., Macchi, M.: A review of the roles of digital twin in CPS-based production systems. Procedia Manufacturing. 11, 939-948 (2017). https://doi.org/10.1016/ j.promfg.2017.07.198

Nyhuis, P., Wiendahl, H.-P.: Fundamentals of Production Logistics: Theory, Tools and Applications. Springer, Berlin (2009)

Ōno, T.: Das Toyota-Produktionssystem. Produktion. Campus-Verl, Frankfurt (2013)

Ouelhadj, D., Petrovic, S.: A survey of dynamic scheduling in manufacturing systems. J. Sched. 12, 417-431 (2009). https://doi.org/10.1007/s10951-008-0090-8

Perkins, C.E.: Ad Hoc Networking: An Introduction. Addison-Wesley, Boston (2001)

Philipp, T.: Messung und Bewertung selbststeuernder Produktionssysteme. Doctoral dissertation, University of Bremen (2014)

Philipp, T., Böse, F., Windt, K.: Autonomously controlled processes-characterisation of complex production systems. In: Proceedings of 3rd CIRP Conference in Digital Enterprise Technology, Setubal, Portugal (2006)

Philipp, T., de Beer, C., Windt, K., Scholz-Reiter, B.: Evaluation of autonomous logistic processes - analysis of the influence of structural complexity. In: Understanding Autonomous Cooperation and Control in Logistics, pp. 303-324. Springer, Berlin, Heidelberg (2007)

Rekersbrink, H., Wenning, B.-L.: Selbststeuerung in der Transportlogistik - Evaluation einer Selbststeuerungsmethode anhand dynamischer Pickup and Delivery Probleme. Industrie Management. 27, 30-34 (2011)

Rekersbrink, H., Ludwig, B., Scholz-Reiter, B.: Entscheidungen selbststeuernder logistischer Objekte. Industrie Management. 23, 25-30 (2007)

Rekersbrink, H., Makuschewitz, T., Scholz-Reiter, B.: A distributed routing concept for vehicle routing problems. Logist. Res. 1, 45-52 (2009). https://doi.org/10.1007/s12159-008-0003-4

Rekersbrink, H., Scholz-Reiter, B., Zabel, C.: An autonomous control concept for production logistics. In: Advanced Manufacturing and Sustainable Logistics, pp. 245-256. Springer, Berlin, Heidelberg (2010)

Scholz-Reiter, B., Jagalski, T.: Dynamics of autonomous control in production logistics. In: 26th International Conference of the System Dynamics Society, p. 15. System Dynamics Society, Albany (2008) 
Scholz-Reiter, B., Freitag, M., de Beer, C., Jagalski, T.: Modelling and analysis of autonomous shop floor control. In: Proceedings of 38th CIRP International Seminar on Manufacturing Systems. Universidade Federal de Santa Catarina, Florianopolis (2005a)

Scholz-Reiter, B., Freitag, M., de Beer, C., Jagalski, T.: Modelling dynamics of autonomous logistic processes: discrete-event versus continuous approaches. CIRP Ann. 54, 413-416 (2005b). https://doi.org/10.1016/s0007-8506(07)60134-6

Scholz-Reiter, B., Rekersbrink, H., Freitag, M.: Kooperierende Routingprotokolle zur Selbststeuerung von Transportprozessen. Industrie Management. 22, 7-10 (2006a)

Scholz-Reiter, B., Freitag, M., de Beer, C., Jagalski, T.: Modelling and simulation of a pheromone based shop floor control. In: Proceedings of the 3rd International CIRP Sponsored Conference on Digital Enterprise Technology, Setubal, pp. 1-7 (2006b)

Scholz-Reiter, B., Freitag, M., de Beer, C., Jagalski, T.: The influence of production networks' complexity on the performance of autonomous control methods. In: Proceedings of the $5^{\text {th }}$ CIRP International Seminar on Computation in Manufacturing Engineering, pp. 317-320. Naples, Italy (2006c)

Scholz-Reiter, B., Jagalski, T., de Beer, C., Freitag, M.: Autonomous shop floor control considering set-up times. In: Proceedings of the 40th CIRP International Seminar on Manufacturing Systems. University of Liverpool, Liverpool (2007a)

Scholz-Reiter, B., Kolditz, J., Hildebrandt, T.: UML as a basis to model autonomous production systems. In: Digital Enterprise Technology, pp. 553-560. Springer, Berlin (2007b)

Scholz-Reiter, B., Jagalski, T., Bendul, J.C.: Autonomous control of a shop floor based on Bee's foraging behaviour. In: Kreowski, H.-J., Scholz-Reiter, B., Haasis, H.-D. (eds.) Dynamics in Logistics, pp. 415-423. Springer, Berlin, Heidelberg (2008a)

Scholz-Reiter, B., de Beer, C., Freitag, M., Jagalski, T.: Bio-inspired and pheromone-based shopfloor control. Int. J. Comput. Integr. Manuf. 21, 201-205 (2008b). https://doi.org/10.1080/ 09511920701607840

Scholz-Reiter, B., Rekersbrink, H., Wenning, B.-L., Makuschewitz, T.: A survey of autonomous control algorithms by means of adapted vehicle routing problems. In: Proceedings of the 9th Biennial ASME Conference on Engineering Systems Design and Analysis, pp. 411-416. American Society of Mechanical Engineers Digital Collection, New York (2009a)

Scholz-Reiter, B., Özsahin, M.E., Teucke, M., Mehrsai, A.: Autonomous order allocation in large distance apparel industry supply chains based on use of RFID technology. In: Proceedings of the $14^{\text {th }}$ International Symposium On Logistics, pp. 329-336. Global Supply Chains and InterFirm Networks, Centre for Concurrent Enterprise Nottingham University Business School, Nottingham (2009b)

Scholz-Reiter, B., Görges, M., Philipp, T.: Autonomously controlled production systems - influence of autonomous control level on logistic performance. CIRP Ann. 58, 395-398 (2009c). https://doi.org/10.1016/j.cirp.2009.03.011

Scholz-Reiter, B., Görges, M., Jagalski, T., Mehrsai, A.: Modelling and analysis of autonomously controlled production networks. IFAC Proc. 42, 846-851 (2009d). https://doi.org/10.3182/ 20090603-3-RU-2001.0081

Scholz-Reiter, B., Rekersbrink, H., Görges, M.: Dynamic flexible flow shop problems - scheduling heuristics vs. autonomous control. CIRP Ann. 59, 465-468 (2010a). https://doi.org/10.1016/ j.cirp.2010.03.030

Scholz-Reiter, B., Zabel, C., Rekersbrink, H.: Influence of competition-based knowledge restrictions on autonomous routing in transport logistics. In: Proceedings of the 2nd International Conference on Logistics and Transport, Queenstown, Neuseeland (2010b)

Scholz-Reiter, B., Görges, M., Jagalski, T., Naujok, L.: Modelling and analysis of an autonomous control method based on bacterial chemotaxis. In: 43rdCIRP International Conference on Manufacturing Systems 2010, pp. 699-706. Neuer Wissenschaftlicher Verlag, Wien (2010c)

Scholz-Reiter, B., Ruthenbeck, C., Teucke, M., Hoppert, J.: Limitations of autonomous control in practical applications: report on lessons learned from vehicle and apparel logistics. In: Autonomous Cooperation and Control in Logistics, pp. 291-311. Springer, Berlin, Heidelberg (2011a) 
Scholz-Reiter, B., Görges, M., Jagalski, T.: Modeling and analyzing integrated autonomously controlled production and transport processes. In: Proceedings of the 21st International Conference On Production Research, vol 21 (2011b)

Scholz-Reiter, B., Görges, M., Rekersbrink, H.: Potentials and limitations of autonomously controlled production systems. In: Autonomous Cooperation and Control in Logistics, pp. 131147. Springer, Berlin, Heidelberg (2011c)

Scholz-Reiter, B., Dashkovskiy, S., Görges, M., Naujok, L.: Stability analysis of autonomously controlled production networks. Int. J. Prod. Res. 49, 4857-4877 (2011d). https://doi.org/ 10.1080/00207543.2010.505215

Scholz-Reiter, B., Werthmann, D., Lappe, D., Otterstedt, N.: Ortungsgestützte Steuerung von Qualitätssicherungsprozessen. Industrie Management. 28, 22-26 (2012)

Schukraft, S., Grundstein, S., Freitag, M., Scholz-Reiter, B.: Strategies for the coupling of autonomous control and central planning: Evaluation of strategies using logistic objectives achievement and planning adherence. In: IEEE International Conference on Systems, Man, and Cybernetics, pp. 121-126. IEEE, Piscataway (2015)

Schukraft, S., Grundstein, S., Scholz-Reiter, B., Freitag, M.: Evaluation approach for the identification of promising methods to couple central planning and autonomous control. Int. J. Comput. Integr. Manuf. 29, 438-461 (2016a). https://doi.org/10.1080/0951192X.2015.1066032

Schukraft, S., Grundstein, S., Freitag, M., Scholz-Reiter, B.: Evaluation of strategies for the coupling of central planning and autonomous control in dynamic job shop environments. Adv. Mater. Res. 1140, 457-464 (2016b). https://doi.org/10.4028/www.scientific.net/ AMR.1140.457

Singh, G., Wenning, B.-L., Görg, C.: Efficient communication in autonomous logistic processes by application of cluster-based routing methods. In: Proceedings of Summer Computer Simulation Conference (2008)

Sowade, S., Rippel, D., Scholz-Reiter, B.: Modeling concept for the infrastructure of autonomous logistic processes. CIRP J. Manuf. Sci. Technol. 5, 254-266 (2012). https://doi.org/10.1016/ j.cirpj.2012.09.011

Teucke, M., Broda, E., Börold, A., Freitag, M.: Using sensor-based quality data in automotive supply chains. Mach. Des. 6, 53 (2018). https://doi.org/10.3390/machines6040053

Thoben, K.-D., Veigt, M., Lappe, D., Franke, M., Kück, M., Kolberg, D., Fahl, I., Zimmerling, R., Schlick, J., Stephan, P., Guth, P.: Towards networking logistics resources to enable a demanddriven material supply for lean production systems - basic concept and potential of a cyberphysical logistics system. In: Proceedings of the 7. BVL Scientific Symposium on Logistics, pp. 42-69. BVL International Bundesvereinigung Logistik (BVL) e.V, Bremen (2014)

University of Bremen.: Bremen Research Cluster for Dynamics in Logistics (LogDynamics). http:/ /www.logdynamics.de/logdynamics.html?\&L=1 (2021). Accessed 18 June 2021

Warden, T., Porzel, R., Gehrke, J.D., Herzog, O., Langer, H., Malaka, R.: Towards ontology-based multiagent simulations: the Plasma approach. In: Bargiela, A. (ed.) 24th European Conference on Modelling and Simulation ECMS 2010: June 1st-4th, 2010, Kuala Lumpur, Malaysia: Proceedings, pp. 50-56. European Council for Modelling and Simulation (ECMS), Dudweiler (2010)

Wenning, B.-L.: Context-Based Routing in Dynamic Networks. Vieweg+Teubner, Wiesbaden (2010)

Wenning, B.-L., Görg, C., Peters, K.: Ereignisdiskrete Modellierung von Selbststeuerung in Transportnetzen. Industrie Management. 21, 53-56 (2005)

Wenning, B.-L., Pesch, D., Timm-Giel, A.: A distributed routing approach for vehicle routing in logistic networks. In: 64th IEEE Vehicular Technology Conference: Montreal, Quebec, Canada. IEEE, Piscataway, NJ (2006)

Wenning, B.-L., Rekersbrink, H., Becker, M., Timm-Giel, A., Görg, C., Scholz-Reiter, B.: Dynamic transport reference scenarios. In: Hülsmann, M., Windt, K. (eds.) Understanding Autonomous Cooperation and Control in Logistics, pp. 337-350. Springer, Berlin, Heidelberg (2007a) 
Wenning, B.-L., Görg, C., Timm-Giel, A., Schönberger, J., Kopfer, H.: Investigations on objectcentered routing in dynamic environments: algorithmic framework and initial numerical results. In: Proceedings of the 9th International Conference on Enterprise Information Systems, pp. 225-230. Madeira, Portugal (2007b)

Wenning, B.-L., Rekersbrink, H., Görg, C.: Scalability investigations on communication traffic in distributed routing of autonomous logistic objects. In: 9th International Conference on Intelligent Transport Systems Telecommunications: Lille, France, pp. 8-12. IEEE, Piscataway, NJ (2009)

Wenning, B.-L., Pesch, D., Timm-Giel, A., Görg, C.: Environmental monitoring aware routing: making environmental sensor networks more robust. Telecommun. Syst. 43, 3-11 (2010). https://doi.org/10.1007/s11235-009-9191-8

Wenning, B.-L., Rekersbrink, H., Timm-Giel, A., Görg, C.: Weighted multiplicative decision function for distributed routing in transport logistics. In: Dynamics in Logistics, pp. 117-124. Springer, Berlin, Heidelberg (2011)

Windt, K., Hülsmann, M.: Changing paradigms in logistics - understanding the shift from conventional control to autonomous cooperation and control. In: Hülsmann, M., Windt, K. (eds.) Understanding Autonomous Cooperation and Control in Logistics, pp. 1-16. Springer, Berlin, Heidelberg (2007)

Windt, K., Philipp, T., Böse, F.: Complexity cube for the characterization of complex production systems. Int. J. Comput. Integr. Manuf. 21, 195-200 (2008). https://doi.org/10.1080/ 09511920701607725

Windt, K., Becker, T., Jeken, O., Gelessus, A.: A classification pattern for autonomous control methods in logistics. Logist. Res. 2, 109-120 (2010a). https://doi.org/10.1007/s12159-0100030-9

Windt, K., Becker, T., Kolev, I.: A comparison of the logistics performance of autonomous control methods in production logistics. In: Proceedings of the 43rd CIRP International Conference on Manufacturing Systems. Sustainable Production and Logistics in Global Networks, pp. 576583. NW Verlag, Vienna (2010b)

Windt, K., Philipp, T., Böse, F., Becker, T.: Application of a three-component evaluation system for autonomous control in logistics. Proc. Inst. Mech. Eng. B J. Eng. Manuf. 224, 1267-1276 (2010c). https://doi.org/10.1243/09544054JEM1656

Open Access This chapter is licensed under the terms of the Creative Commons Attribution 4.0 International License (http://creativecommons.org/licenses/by/4.0/), which permits use, sharing, adaptation, distribution and reproduction in any medium or format, as long as you give appropriate credit to the original author(s) and the source, provide a link to the Creative Commons license and indicate if changes were made.

The images or other third party material in this chapter are included in the chapter's Creative Commons license, unless indicated otherwise in a credit line to the material. If material is not included in the chapter's Creative Commons license and your intended use is not permitted by statutory regulation or exceeds the permitted use, you will need to obtain permission directly from the copyright holder. 